\title{
Delaunay Graphs Are Almost as Good as Complete Graphs*
}

David P. Dobkin, Steven J. Friedman, and Kenneth J. Supowit

Department of Computer Science, Princeton University, Princeton, NJ 08544, USA

Abstract. Let $S$ be any set of $N$ points in the plane and let DT( $S)$ be the graph of the Delaunay triangulation of $S$. For all points $a$ and $b$ of $S$, let $d(a, b)$ be the Euclidean distance from $a$ to $b$ and let $\operatorname{DT}(a, b)$ be the length of the shortest path in $\mathrm{DT}(S)$ from $a$ to $b$. We show that there is a constant $c(\leq((1+\sqrt{5}) / 2) \pi \approx 5.08)$ independent of $S$ and $N$ such that

$$
\frac{\mathrm{DT}(a, b)}{d(a, b)}<c
$$

\section{Introduction}

Let $\mathrm{DL}_{i}(S)$ be the Delaunay triangulation of $S$ in the $L_{i}$ norm $(i=1,2)$. Chew [Ch] shows that there exists a constant $c_{1}$ such that the ratio of shortest distances in $\mathrm{DL}_{1}(S)$ to straight line (i.e., $L_{2}$ ) distances is bounded above by $c_{1}$ where $c_{1}=\sqrt{10} \approx 3.16228$. We extend this result here demonstrating a constant $c_{2}$ such that the ratio of shortest distances in $\mathrm{DL}_{2}(S)$ to straight line distances is bounded above by $c_{2}=((1+\sqrt{5}) / 2) \pi \approx 5.08$. The best-known lower bound on $c_{2}$ is $\pi / 2$ and is also due to Chew.

In his paper, Chew describes applications of his (and our) result to problems of motion planning, polygon visibility, and extensions of Voronoi diagrams/Delaunay triangulations. Our focus is the derivation of $c_{2}$ and potential extensions to other problems involving distances in the plane.

In what follows, we provide the definitions and lemmas necessary to prove our main result in Section 2; Section 3 contains the proofs. We conclude with some open problems.

* This research was supported in part by an AT\&T Bell Laboratories Scholarship, by NSF Grants DMC-8451214, CCR87-00917, and CCR85-05517, and by a grant from the IBM Corporation. 


\section{The Main Result}

We begin with (informal) definitions of the Voronoi diagram and the Delaunay triangulation. The Voronoi diagram for a set $S$ of $N$ points in the plane is a partition of the plane into regions, each containing exactly one point in $S$, such that, for each point $p \in S$, every point within its corresponding region (denoted $\operatorname{Vor}(p))$ is closer to $p$ than to any other point of $S$. The boundaries of these regions form a planar graph. The Delaunay triangulation of $S$ is the straight-line dual of the Voronoi diagram for $S$; that is, we connect a pair of points in $S$ if and only if they share a Voronoi boundary. Under the standard assumption that no four points of $S$ are cocircular, the Delaunay triangulation is indeed a triangulation [PS]; we denote its corresponding graph by DT $(S)$.

For the remainder of this section, fix points $a, b \in S$; we will construct a path in $\mathrm{DT}(S)$ that is not too long in relation to $d(a, b)$. Assume for simplicity that $a$ and $b$ lie on the $x$-axis, with $x(a)<x(b)$ (we denote the coordinates of a point $q$ in the plane by $x(q)$ and $y(q)$, respectively). We refer to members of $S$ alternatively as points or vertices, and to edges of DT $(S)$ as edges or line segments, as the context indicates.

Our original idea for the path was simply to use the vertices $a=b_{0}$, $b_{1}, \ldots, b_{m-1}, b_{m}=b$ corresponding to the sequence of Voronoi regions traversed by walking from $a$ to $b$ along the $x$-axis, as illustrated in Fig. 1, where $m=4$ (in the case in which a Voronoi edge happens to lie on the $x$-axis somewhere between $a$ and $b$, we-arbitrarily-choose that Voronoi region lying above, rather than

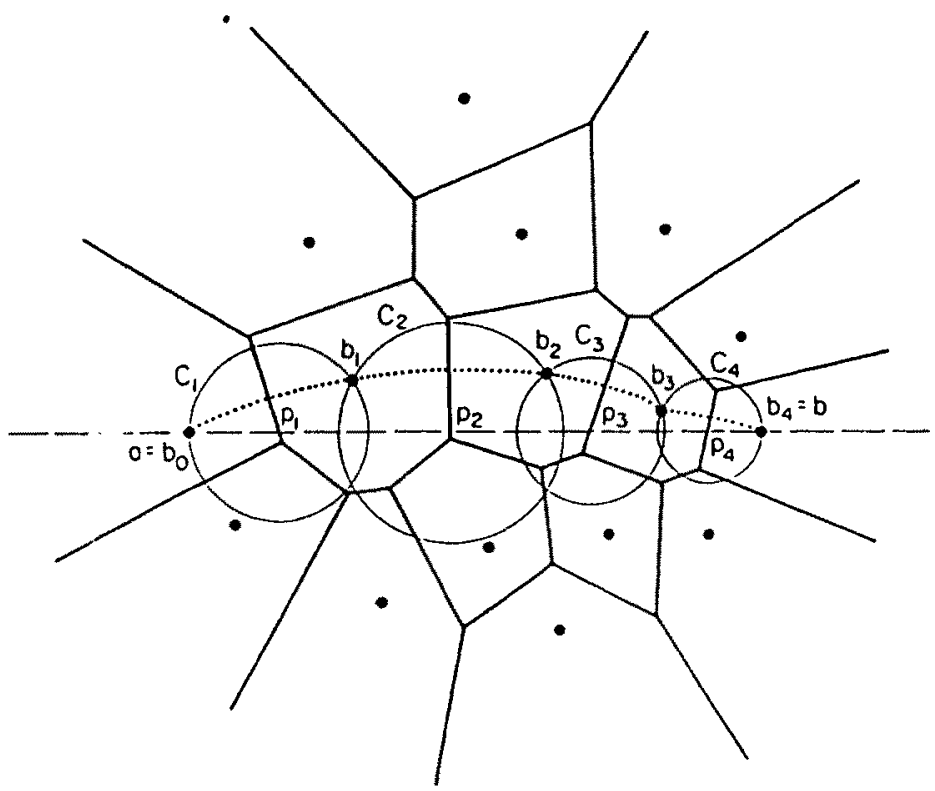

Fig. 1. The Voronoi diagram is shown in solid line, and the direct DT path between $a$ and $b$ in dotted line. 
below, the $x$-axis). In general, we refer to the DT path constructed in this way between some $z$ and $z^{\prime}$ in $S$ as the direct DT path from $z$ to $z^{\prime}$. Let $p_{i}$ denote the point on the $x$-axis that also lies on the boundary between $\operatorname{Vor}\left(b_{i-1}\right)$ and $\operatorname{Vor}\left(b_{i}\right)$, for $i=1,2, \ldots, m$. The definition of the Voronoi diagram immediately gives that $p_{i}$ is the center of a circle $C_{i}$ passing through $b_{i-1}$ and $b_{i}$ but containing no points of $S$ in its interior.

Two simple properties of direct DT paths are:

Lemma 1. $x\left(b_{0}\right) \leq x\left(b_{1}\right) \leq \cdots \leq x\left(b_{m}\right)$.

Lemma 2. For all $i, 0 \leq i \leq m, b_{i}$ is contained within, or on the boundary of, circle $(a, b)$ (by which we denote the circle with $a$ and $b$ diametrically opposed).

Note in Fig. 1 that all the $b_{i}$ happen to be in the same half-plane defined by the line connecting $a$ and $b$ (i.e., $y\left(b_{i}\right) \geq 0$ for all $0 \leq i \leq m$ ). In such cases, we say that the direct path between the two points is one-sided. One-sided paths are fortuitous for our purposes, because the ratio of the path length to the Euclidean distance is at most $\pi / 2$; this is a simple consequence of Lemma 1 above and the following:

Lemma 3. Let $D_{1}, D_{2}, \ldots, D_{k}$ be circles all centered on the $x$-axis such that $D=\bigcup_{1 \leq i \leq k} D_{i}$ is connected. Then boundary $(D)$ has length at most $\pi \cdot\left(x_{r}-x_{1}\right)$, where $x_{1}$ and $x_{r}$ are the least and greatest $x$-coordinates of $D$, respectively (see Fig. 2).

Lemma 3 applies to the one-sided paths because the half of boundary $(C)$ (where $C$ is defined as $\bigcup_{1 \leq k \leq m} C_{k}$ ) that lies above the $x$-axis has length at least as great as the path itself (because the $b_{i}$ are monotonic in $x$ ).

The trouble with this approach is that the path is not necessarily even close to being one-sided; the path may zig-zag across the $x$-axis (as is illustrated in Fig. 3) $\Theta(N)$ times.

Our modified approach, then, is to try to stay above the $x$-axis. Should the direct path dip below the $x$-axis, we determine how costly the dip will be. If dipping below is not too expensive (in a sense defined below) then we follow the direct path below the $x$-axis and then back up. Otherwise, we construct a shortcut between the two points above the $x$-axis. Most of the proof consists of showing that the shortcut is not too long. The exact path we take is made more precise in the proof of the following:

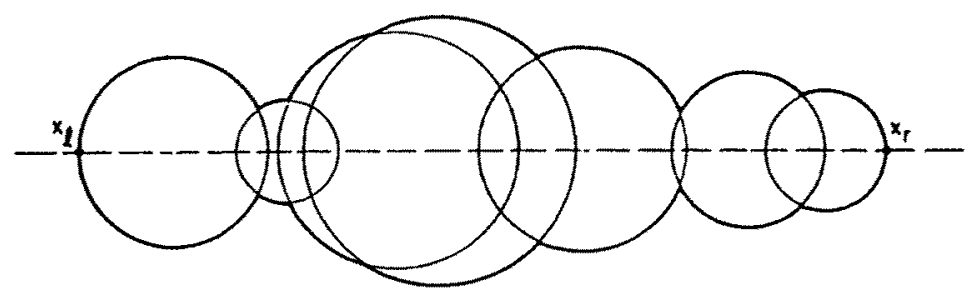

Fig. 2. Illustration for Lemma 3. 


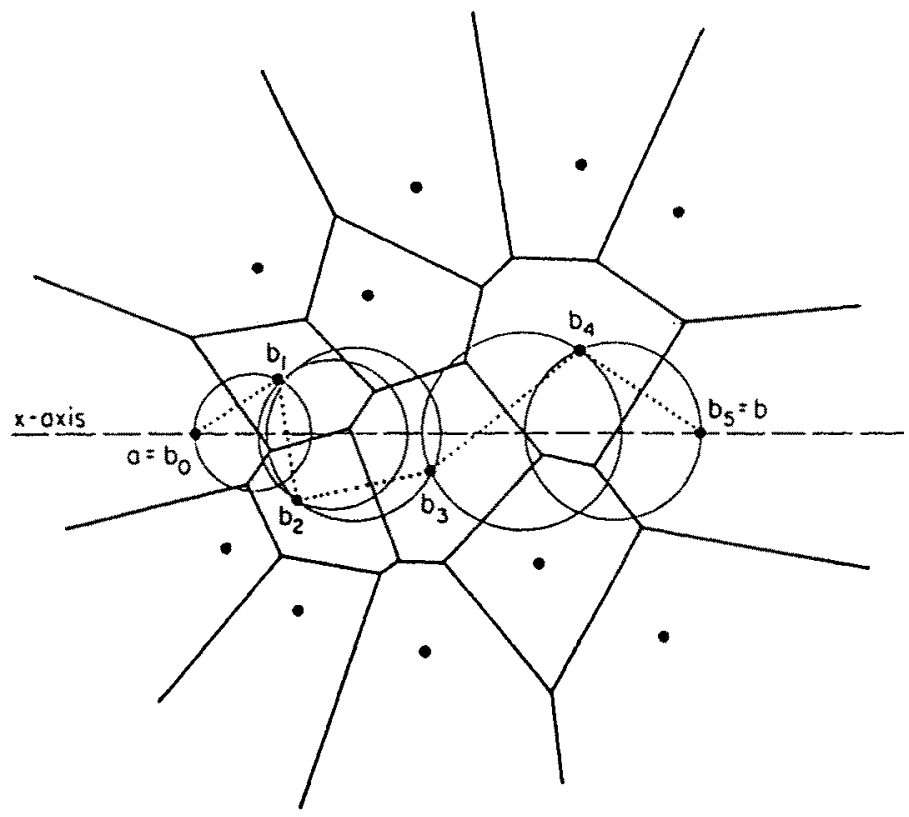

Fig. 3. A direct DT path that is not one-sided.

Theorem. There exists a DT path from $a$ to $b$ of length

$$
\leq((1+\sqrt{5}) / 2) \pi \cdot d(a, b) \text {. }
$$

Proof. We present an algorithm for constructing a DT path from $a=b_{0}$ to $b=b_{m}$, and then analyze the length of the path it produces. Assume that the path so far has brought us to some $b_{i}$ such that (1) $y\left(b_{i}\right) \geq 0$ (initially, $\left.i=0\right),(2) i<m$ (meaning we are not finished), and (3) $y\left(b_{i+1}\right)<0$. Thus the direct path would dip below the $x$-axis for a while after $b_{i}$. Let $j$ be the least number greater than $i$ such that $y\left(b_{j}\right) \geq 0$ (e.g., in Fig. 4, if $i=2$ then $j=4$ ). Let $T$ denote the path along the boundary of $C$ clockwise from $b_{i}$ to $b_{j}$. Let $w$ denote the length of the projection of $T$ onto the $x$-axis (thus $w=x\left(b_{j}\right)-x\left(b_{i}\right)$ ). Define $h=$ $\min \{y(q): q$ lies on $T\}$. Now if $h \leq w / 4$ then continue along the direct path to $b_{j}$

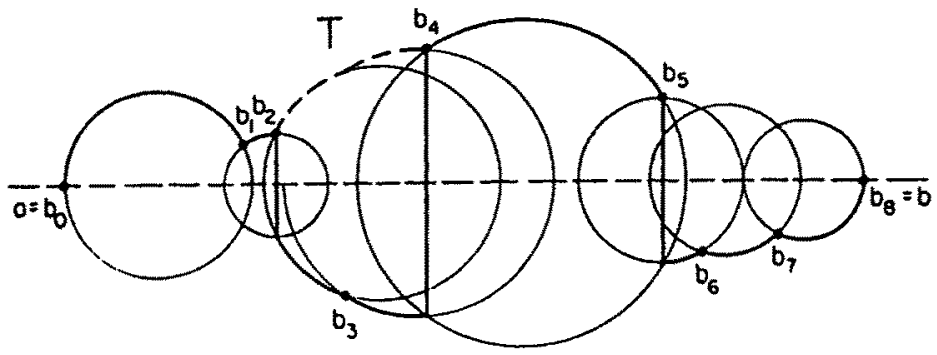

Fig. 4. An upper bound on the length of the direct DT path. 


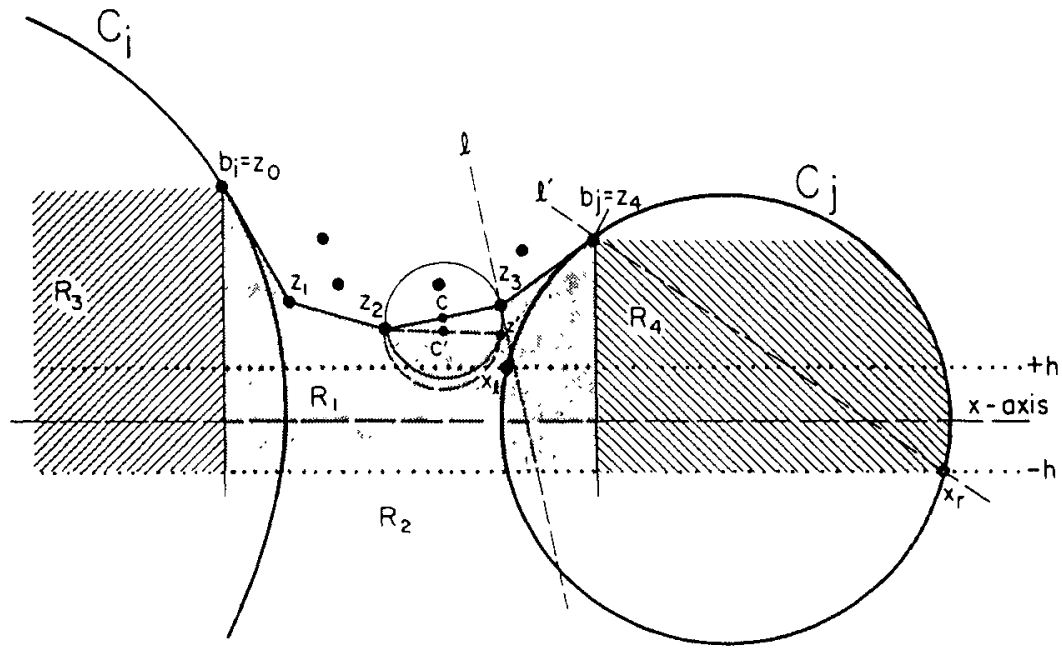

Fig. 5. The shortcut from $b_{1}$ to $b_{j}$. Here $k=2$.

(i.e., use edges $b_{i} b_{i+1}, b_{i+1} b_{1+2}, \ldots, b_{j-1} b_{j}$ ). Otherwise we take a shortcut as follows. Construct the lower convex hull $b_{i}=z_{0}, z_{1}, z_{2}, \ldots, z_{n}=b_{j}$ of the set

$$
\left\{q \in S: x\left(b_{i}\right) \leq x(q) \leq x\left(b_{j}\right) \text { and } y(q) \geq 0 \text { and } q \text { lies under } b_{i} b_{j}\right\}
$$

(see Fig. 5). Note that these convex hull edges are certainly not on the direct DT path from $a$ to $b$. Now the shortcut consists of taking the direct DT path from $z_{k}$ to $z_{k+1}$ for each $0 \leq k \leq n-1$. The key fact (proved in Section 3 ) is:

Lemma 4. Let $z_{k} z_{k+1}$ be an edge of the lower convex hull described above. Then the direct $D T$ path from $z_{k}$ to $z_{k+1}$ is one-sided.

Next we analyze the length of the path produced by this algorithm. When proceeding from $b_{i}$ to $b_{j}$, let $t$ denote the length of $T$. If $h \leq w / 4$ then let $q_{0}$ be the point of $T$ with least $y$-value (see Fig. 6), let $t_{i}$ denote the length of the portion of $T$ from $b_{i}$ to $q_{0}$, and $t_{j}$ the length of the portion of $T$ from $q_{0}$ to $b_{j}$ (thus $\left.t_{i}+t_{j}=t\right)$. Let $w_{i}$ and $w_{j}$ denote the lengths of the projections of those two portions of $T$, respectively (thus $w_{i}+w_{j}=w$ ). Then the path we take (i.e., no shortcuts) has length at most

$$
\begin{aligned}
t+2\left(y\left(b_{i}\right)+y\left(b_{j}\right)\right) & =t+2\left(2 h+\left(y\left(b_{i}\right)-h\right)+\left(y\left(b_{j}\right)-h\right)\right) \\
& \leq t+2\left(\frac{w}{2}+\left(y\left(b_{i}\right)-h\right)+\left(y\left(b_{j}\right)-h\right)\right) \\
& =t+2\left(\frac{w_{i}}{2}+\left(y\left(b_{i}\right)-h\right)+\frac{w_{j}}{2}+\left(y\left(b_{j}\right)-h\right)\right) \\
& \leq t+2\left(\frac{\sqrt{5}}{2} t_{i}+\frac{\sqrt{5}}{2} t_{j}\right)=t(1+\sqrt{5}) .
\end{aligned}
$$




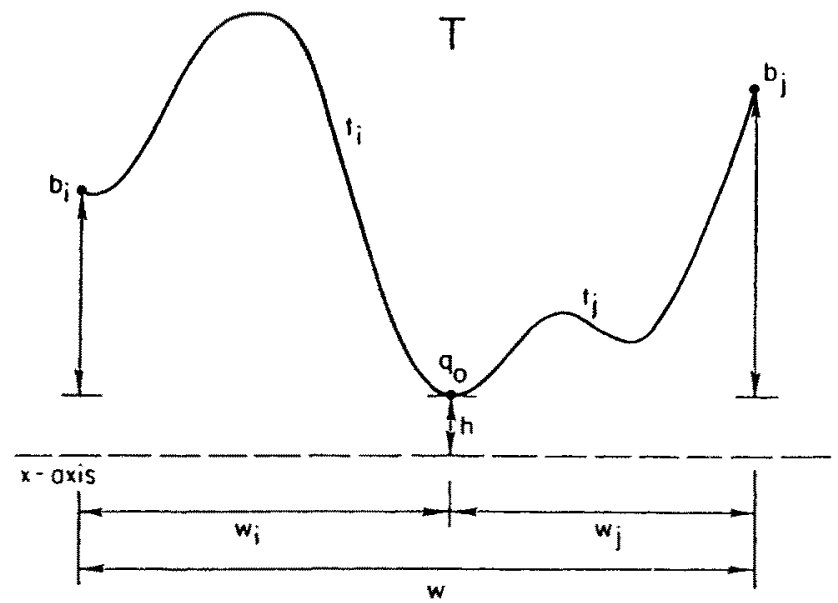

Fig. 6. Analyzing the path length when the shortcut is not taken.

The last inequality follows from the (easily proved) fact that

$$
\frac{a}{2}+b \leq \frac{\sqrt{5}}{2} c
$$

whenever $a$ and $b$ are the legs of a right triangle with hypotenuse $c$.

On the other hand, if $h>w / 4$ then we take the shortcut, which has length at most

$$
\sum_{k=0}^{n-1} \text { length of one-sided path from } z_{k} \text { to } z_{k+1}
$$

(by Lemma 4) which is $\leq \sum_{k=0}^{n-1} d\left(z_{k}, z_{k-1}\right) \pi / 2 \leq t \pi / 2$ (by Lemma 3). Hence in either case, the distance we travel in getting from $b_{i}$ to $b_{j}$ is at most $(1+\sqrt{5}) t$. Therefore summing over all such trips $b_{i}$ to $b_{j}$ as well as the trips (for which we travel at most $t$ units) where the direct DT path from $a$ to $b$ stays completely above the $x$-axis, we get (by Lemma 3 ) a total path length of at most $d(a, b)((1+\sqrt{5}) / 2) \pi$.

\section{Proofs of the Lemmas}

Proof of Lemma 1. The perpendicular bisector of $b_{i}$ and $b_{i+1}$ contains $p_{i}$. Point $b_{i+1}$ lies to the right of this bisector, and $b_{i}$ lies to the left; hence $x\left(b_{i}\right) \leq$ $x\left(b_{i+1}\right)$.

Proof of Lemma 2. Let $c$ denote the midpoint of segment $a b$; let $k$ be such that $c$ lies in the Voronoi region of $b_{k}$. Then

$$
d\left(b_{0}, c\right) \geq d\left(b_{1}, c\right) \geq \cdots \geq d\left(b_{k}, c\right)
$$




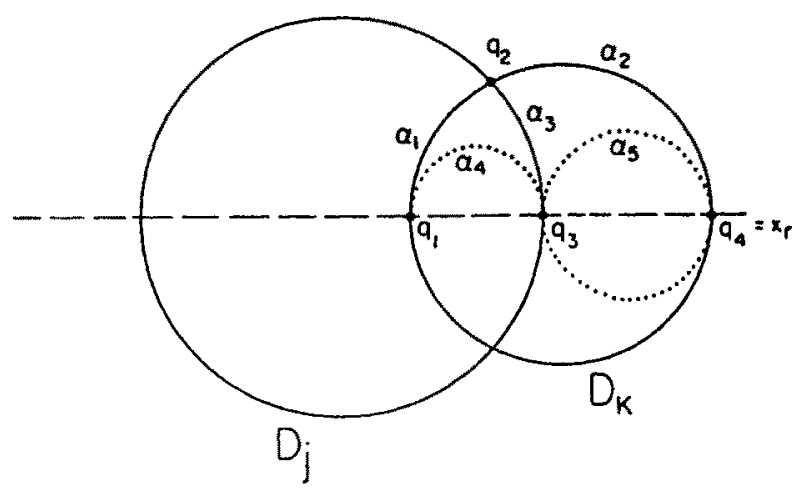

Fig. 7. Illustration for the proof of Lemma 3.

and

$$
d\left(b_{k}, c\right) \leq d\left(b_{k+1}, c\right) \leq \cdots \leq d\left(b_{m}, c\right) \text {. }
$$

Proof of Lemma 3. By induction on $k$. The claim is easy if $k=1$; so let $k \geq 2$ and assume it for $k-1$. Let $q_{1}$ and $q_{4}$ denote the leftmost and rightmost points of $D_{k}$, respectively (see Fig. 7), and assume without loss of generality that $q_{4}=x_{r}$. Let $q_{2}$ be the rightmost point at which $D_{k}$ intersects another circle $D_{j}$ (thus $j<k$ ); let $q_{3}$ be the rightmost point of $D_{j}$. We can assume that $D_{k}$ does not entirely contain any circle $D_{i}(i \neq k)$, since otherwise $D_{i}$ would not contribute to boundary $(D)$ and hence the induction would be trivial. Denote by $\alpha_{1}\left(\alpha_{2}\right)$ the length of the arc on circle $D_{k}$ clockwise from $q_{1}$ to $q_{2}$ (resp. $q_{2}$ to $q_{4}$ ). Let $\alpha_{3}$ be the length of the arc on circle $D_{j}$ clockwise from $q_{2}$ to $q_{3}$. Finally, let $\alpha_{4}=$ $(\pi / 2)\left(x\left(q_{3}\right)-x\left(q_{1}\right)\right)$ and let $\alpha_{5}=(\pi / 2)\left(x\left(q_{4}\right)-x\left(q_{3}\right)\right)$. Then a simple convexity argument shows that

$$
\alpha_{1}+\alpha_{3} \geq \alpha_{4}
$$

Also, we have

$$
\alpha_{4}+\alpha_{5}=\alpha_{1}+\alpha_{2}
$$

Hence

$$
\alpha_{1}+\alpha_{3}+\alpha_{5} \geq \alpha_{4}+\alpha_{5}=\alpha_{1}+\alpha_{2},
$$

implying $\alpha_{3}+\alpha_{5} \geq \alpha_{2}$. Therefore, denoting the length of the boundary of $D$ by bd $(D)$, we have

$$
\begin{aligned}
\operatorname{bd}(D) & \leq \operatorname{bd}\left(\operatorname{circle}\left(q_{3}, q_{4}\right) \cup \bigcup_{1 \leq i \leq k-1} D_{i}\right) \\
& \leq \operatorname{bd}\left(\operatorname{circle}\left(q_{3}, q_{4}\right)\right)+\operatorname{bd}\left(\bigcup_{i \leq i \leq k-1} D_{i}\right) \\
& \leq \pi\left(x_{r}-x\left(q_{3}\right)\right)+\pi\left(x\left(q_{3}\right)-x_{1}\right) \quad \text { (by the inductive hypothesis) } \\
& \leq \pi\left(x_{r}-x_{1}\right) .
\end{aligned}
$$


Proof of Lemma 4. By Lemma 2, the direct DT path from $z_{k}$ to $z_{k+1}$ lies entirely within circle $\left(z_{k}, z_{k+1}\right)$. We now show that there are no points of $S$ within the lower semicircle of $\operatorname{circle}\left(z_{k}, z_{k+1}\right)$, so the path must be one-sided.

Let $q$ be an arbitrary point in this lower semicircle; we must show $q \notin S$. If $x\left(b_{i}\right) \leq x(q) \leq x\left(b_{j}\right)$ and $y(q) \geq-h$ (i.e., $q$ lies in region $R_{1}$ in Fig. 5) then we claim $q \notin S$. To see this, note that if $y(q) \geq h$ then it lies outside the lower convex hull; whereas if $-h<y(q)<h$ then $q$ lies in the interior of $\bigcup_{i \leq k \leq j} C_{k}$.

We next show that $y(q)>-h$ (that is, $q \notin R_{2}$ ). Assume without loss of generality that $y\left(z_{k}\right) \leq y\left(z_{k+1}\right)$. Since $z_{k} \in S$ it must lie directly above some point of $T$, since the area below $T$ and above the $x$-axis is contained in $C$ and therefore contains no members of $S$. Therefore $y\left(z_{k}\right) \geq h>w / 4$. Let $z^{\prime}$ be the point with coordinates $\left(x\left(z_{k+1}\right), y\left(z_{k}\right)\right)$. Let $c$ and $c^{\prime}$ denote the midpoints of segments $z_{k} z_{k+1}$ and $z_{k} z^{\prime}$, respectively. Then $y\left(c^{\prime}\right)>w / 4$. That $q \in \operatorname{circle}\left(z_{k}, z^{\prime}\right)$ follows from $q \in$ circle $\left(z_{k}, z_{k+1}\right)$ and $y(q) \leq y\left(z_{k}\right)=y\left(z^{\prime}\right)$. Furthermore, $x\left(z_{k+1}\right)-x\left(z_{k}\right) \leq w$, since by extending $z_{k} z_{k+1}$ on both sides we encounter points on $T$ and since $T$ is connected (and hence the projection of $T$ onto the $x$-axis is at least as long as the projection of $z_{k} z_{k+1}$ onto the $x$-axis). Therefore $\operatorname{radius}\left(\operatorname{circle}\left(z_{k}, z^{\prime}\right)\right) \leq w / 2$. Hence

$$
y(q) \geq y\left(c^{\prime}\right)-\operatorname{radius}\left(\operatorname{circle}\left(z_{k}, z^{\prime}\right)\right)>w / 4-w / 2=-w / 4
$$

Note that $x(q) \geq x\left(b_{i}\right)$ (that is $q \notin R_{3}$ ), because of our assumption $y\left(z_{k}\right) \leq$ $y\left(z_{k+1}\right)$.

Finally, we assume $x(q)>x\left(b_{j}\right)$ (hence $q \in R_{4}$ ). We show that $q$ lies in the interior of $C_{j}$, implying $q \notin S$. Let $x_{1}$ be the leftmost point of intersection of circle $C_{j}$ with the line $y=h$. Let $x_{\mathrm{r}}$ be the rightmost point of intersection of $C_{j}$ with the line $y=-h$. Let $l$ denote the line that passes through $z_{k+1}$ perpendicular to segment $z_{k} z_{k+1}$, and let $l^{\prime}$ be the line containing $b_{j}$ and $x_{r}$. Note that both $l$ and $l^{\prime}$ must have negative slopes. Clearly, the entire circle $\left(z_{k}, z_{k+1}\right)$ lies below $l$ and in particular so does $q$. We claim that this implies that $q$ lies below $l^{\prime}$ as well. To see this, first note that our assumption $y\left(z_{k}\right) \leq y\left(z_{k+1}\right)$ implies $y\left(z_{k+1}\right) \leq y\left(b_{j}\right)$, and hence line $l$ intersects the line $x=x\left(b_{j}\right)$ below $b_{j}$. Therefore it suffices to show that slope $(l) \leq \operatorname{slope}\left(l^{\prime}\right)$ (recall that both are negative). The monotonicity of slopes in the lower convex hull gives $\operatorname{slope}\left(z_{k} z_{k+1}\right) \leq \operatorname{slope}\left(x_{1} b_{j}\right)$. Therefore since $l$ and $l^{\prime}$ are perpendicular to $z_{k} z_{k+1}$ and $x_{1} b_{j}$, respectively (the latter is because $x_{1}$ and $x_{\mathrm{r}}$ are diametrically opposed on $\left.C_{j}\right)$, we have slope $(l) \leq \operatorname{slope}\left(l^{\prime}\right)$. Thus $q$ indeed lies below $l^{\prime}$; hence since $q$ is in $R_{4}$ it must also be in $C_{j}$ and therefore not in $S$.

\section{Related Problems}

There are many interesting problems related to that solved here. For example, Raghavan [Ra] suggests that our results extend to a special case problem in 3-space. He conjectures that if $S$ is a set of points on the unit sphere, there is a 
constant $c$ such that

$$
\frac{d_{H}(a, b)}{d(a, b)}<c
$$

where $d_{H}$ is the distance along edges of the convex hull and $d$ is the (threedimensional) Euclidean distance.

The generalization of our result to arbitrary point sets in 3-space and their Delaunay graphs remains open.

In another direction, Feder and others [Fe] have shown that for each $k \geq 7$ there is a constant $c$ such that, for each finite set $S$ of points in the plane, there is a graph $G$ with vertices corresponding to these points, and the following properties:

(1) Each vertex in $G$ has degree at most $k$.

(2) $d_{G}(a, b) / d(a, b)<c$, where $d_{G}$ is the distance along edges of $G$.

Extensions to the cases $k=5$ and 6 have been proposed by others. It is not difficult to show that no such constant exists for $k=2$. What is the minimum $k$ for which such a result is possible?

\section{Acknowledgment}

The authors thank Arthur Watson for his useful comments during this research.

\section{References}

[Ch] P. Chew, There is a planar graph almost as good as the complete graph, Proceedings of the Second Symposium on Computational Geometry, Yorktown Heights, NY, 1986, pp. 169-177.

[Fe] T. Feder, personal communication, 1988.

[PS] F. P. Preparata and M. I. Shamos, Computational Geometry: An Introduction, Springer-Verlag, New York, 1985.

[Ra] P. Raghavan, personal communication, 1987.

Received October 28, 1987, and in revised form April 8, 1988. 